\title{
PHARMACOLOGICAL PATTERNS IN SUBCORTICAL REINFORCEMENT BEHAVIOR*
}

\author{
M. E. Olds and J. Olds \\ Department of Psychology, The University of Michigan, \\ Ann Arbor, Michigan \\ (Received 21 September 1963)
}

\begin{abstract}
Summary-By means of intercurrent hypothalamic positive reinforcement and tegmental negative reinforcement tests, drug actions on two different brain-stimulated behaviors were assessed simultaneously. Chlorpromazine at $2 \mathrm{mg} / \mathrm{kg}$ was effective in antagonizing both hypothalamic positive reinforcement behavior and tegmental negative reinforcement behavior, but it regularly caused a greater percentage deficit in hypothalamic positive behavior. Meprobamate at $100 \mathrm{mg} / \mathrm{kg}$ did not regularly produce lasting reduction of hypothalamic positive reinforcement behavior; however, it did produce sustained reduction of tegmental negative reinforcement behavior. In the case of meprobamate, therefore, there was regularly a greater percentage deficit in tegmental negative behavior. While the imbalanced action of chlorpromazine against positive reinforcement may be explained by aspects of the testing procedure (such as the widely different speeds of the two behaviors) and have no relation to the difference in emotional sign, the opposite imbalanced action of meprobamate makes it quite clear that the two chemicals have quite different modes of action in relation to these brain and behavior tests. The findings with LSD and amphetamine were less informative; LSD had regularly a brief suppressing effect on hypothalamic behavior; amphetamine augmented the slow tegmenta! negative behavior but often slowed the rapid hypothalamic positive behavior.
\end{abstract}

\section{INTRODUCTION}

ELECTRICAL stimulation in many parts of dorsal tegmentum and thalamus yields aversive effects, that is, negative reinforcement behavior (Delgado et al., 1954). But in many parts of hypothalamus, and particularly in lateral hypothalamus, such stimulation produces rewarding effects, that is, positive reinforcement behavior (OLDS and MILNER, 1954; OLDS and Olds, 1963). Previous studies (Olds, 1959; Olds and Travis, 1960) have shown differences between chlorpromazine and meprobamate in relation to these centrally aroused reinforcement behaviors. Chlorpromazine caused depression of positive reinforcement in doses that spared negative reinforcement. Meprobamate, on the other hand, suppressed the negative reinforcement behavior in doses which spared the positive behavior. In these experiments, the positive reinforcement was produced by stimulating hypothalamus in one group of animals; the negative reinforcement was produced by stimulating tegmentum in a different group.

It appeared possible that the different actions might have resulted from differences between the groups of animals rather than from differences in the effects of the drugs on different brain areas or on different reinforcement functions. The possibility of differences between groups arose from three sources. First, the two groups had different pretraining, one being trained on the positive reinforcement problem with hypothalamic

*This work was supported by grants from the USPHS and from Wallace Laboratories. The authors are grateful to G. BALDRIGHI and D. HoGBERG for skilled technical assistance. 
stimulation, the other being trained on the negative reinforcement problem with tegmental stimulation. Second, the two groups had different electric brain stimulation during tests, and there is a likelihood that electric stimulation in a given brain structure during the course of action of a drug might counteract or potentiate the drug effect. Third, large individual differences in dose sensitivity in rats appear regularly.

In order to inquire further into the nature of the differences observed, the animals of the present study were implanted with two sets of bipolar electrodes so that positive and negative reinforcement tests could be made at the same time and on the same animals. The conditions of pretraining and stimulation during drug tests were thus rendered nearly identical for positive and negative reinforcement behavior. Each dose was given to an animal only once and its effects on intercurrent hypothalamic approach and tegmental escape behavior were measured. Assessment was also made of the effects of a given dose as a function of time after administration.

\section{METHODS \\ The First Experiment}

\section{THE FIRST GROUP}

Five male, albino rats weighing $300-400 \mathrm{~g}$ were used in the first group. The animals were selected from a larger group after surgery and after pretesting on the basis of behavioral screening procedures. Two electrode pairs were implanted in each rat. The surgical methods have been described previously (OLDS and MILNER, 1954). One set of bipolar electrodes was aimed at the lateral hypothalamus, the other at the tegmentum (Figs. 1 and 2). Stereotaxic coordinates were selected with a view to having one pair of electrodes in a "positive reinforcement" area of hypothalamus and one pair in a "negative reinforcement" area of tegmentum (OLDS and OLDS, 1963). The electrodes were bipolar, twisted silver wires, $0.01 \mathrm{in}$. in diameter and insulated except for the cross-section of the tips. Sine-wave stimuli of $60 \mathrm{c} / \mathrm{s}$ were used. Stimulus intensity for a given electrode pair was constant for a test series. For the five hypothalamic electrode pairs, the current settings were selected to produce optimal self-stimulation; they ranged from 40 to $60 \mu \mathrm{A}$. For the five tegmental electrodes the current settings selected to produce optimal escape behavior ranged from 18 to $70 \mu \mathrm{A}$.

\section{Behavioral Methods}

Animals were trained and tested in a wooden chamber $15 \times 10 \times 12$ in. into which two metal levers protruded. This apparatus has been described previously (OLDS, 1960; OLDS and OLDS, 1962). One lever delivered the stimulus for the approach test, the other lever terminated the stimulus for the escape test. Four wires from the two electrode pairs passed through the top of the box, and were connected to a programmed stimulator. An automatic timer changed programs every $2 \mathrm{~min}$. The programs were as follows:

Program 1. The approach test with hypothalamic electrodes was made during the first 2-min period in the box. The left lever, upon depression, caused a $1 / 4-\mathrm{sec}$ train of stimulation via the hypothalamic electrodes; no stimulation was applied unless the lever was depressed. The other lever was inactivated during this interval.

Program 2. An approach test with tegmental electrodes was made during the second 2 -min period. This test was identical to that in Program 1 except that the right lever and the tegmental electrodes were used instead of the left lever and the hypothalamic electrodes. 


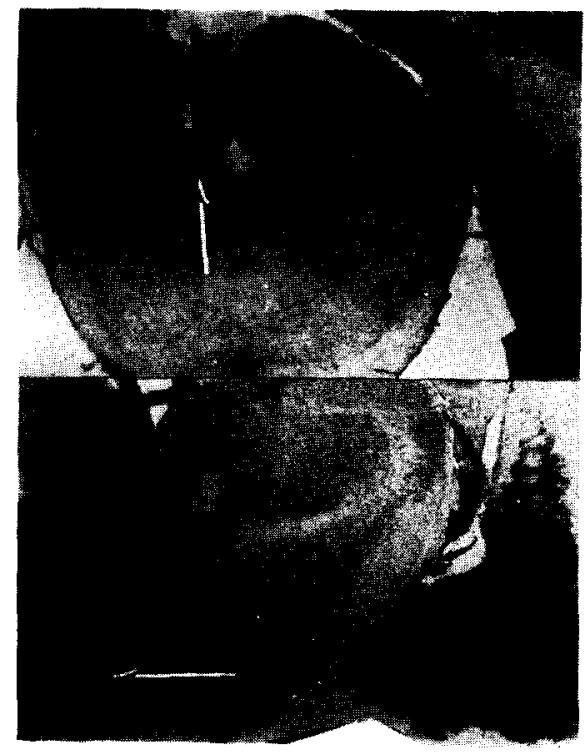

FIG. 1. Enlarged photographs of transverse brain sections showing location of electrode tracks. Upper photo shows path of electrodes yielding escape behavior in animal No. 3468 ; the electrodes stimulated in dorsomedial tegmentum. Lower photo shows path of electrodes yielding positive reinforcement behavior in the same animal; this pair stimulated in lateral hypothalamus. The placements were approximately the same in animals No. 4101, 2991, and 3471 ; No. 3319 had a similarly placed hypothalamic electrode pair.

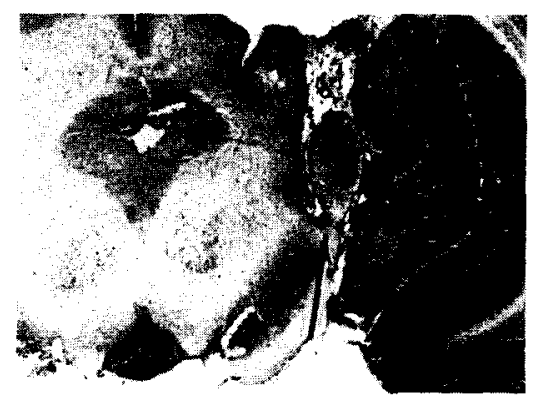

Fici. 2. Path of neutral electrodes which stimulated in a lateral region of brain stem betweên medial geniculate and substantia nigra in animal No. 3319. 
